\title{
Kinetic thin current sheets: their formation in relation to magnetotail mesoscale turbulent dynamics
}

\author{
A. P. Kropotkin and V. I. Domrin \\ Skobeltsyn Institute of Nuclear Physics, Moscow State University, 119992 Moscow, Russia \\ Received: 11 August 2008 - Revised: 28 October 2008 - Accepted: 12 November 2008 - Published: 1 April 2009
}

\begin{abstract}
Dynamics of the magnetotail plasma sheet (PS) features nonlinear structures on two totally different scales. There are very thin current sheets (CS) on kinetic scale of the ion gyroradius. And there are intense plasma flow and magnetic field variations on mesoscales (a few earth radii); those are interpreted as mostly 2-D MHD turbulence. On the other hand, the specific nature of slow large scale magnetotail evolution leads to large differences in the PS properties and those of the lobe plasma. As a result, while fast reconnection bursts in the tail provide quasi-stationary fast mesoscale reconfigurations in the lobes, they cannot however be accompanied by restructuring of CS on the same fast time scale. Violations of force balance in the PS are thus generated. Simulation using a hybrid code and starting with such imbalance, provides an evidence of very thin kinetic CS structures formation, embedded into the much thicker PS. The momentum balance gets locally restored by means of ion acceleration up to the Alfvénic velocity. The process provides an effective mechanism for transformation of magnetic energy accumulated in the magnetotail, into energy of plasma flows. The fast flows may drive turbulence on shorter spatial scales. In their turn, these motions may serve as an origin for new neutral line generation, and reconnection. Application to substorm phenomenology is discussed.
\end{abstract}

Keywords. Magnetospheric physics (Magnetospheric configuration and dynamics; Magnetotail; Plasma sheet; Storms and substorms)

\section{Introduction}

Recent satellite observations, especially those onboard CLUSTER, have revealed a novel pattern of the magnetotail plasma sheet structure and behavior. It appears that in-

Correspondence to: A. P. Kropotkin (apkrop@dec1.sinp.msu.ru) stead of a more or less quiescent global structure and behavior, interrupted from time to time by neutral line formation and magnetic reconnection, intense nonlinear disturbances, at various space and time scales, exist in the plasma sheet permanently. Their most remarkable features are (a) plasma flows and magnetic field variations interpreted as "MHD turbulence" (e.g. Borovsky and Funsten, 2003; Weygand et al., 2005), and (b) sporadic thin "current sheets" (CS) embedded in the plasma sheet (e.g. Runov et al., 2006). The questions appear: Is there any inter-relation of these features? If yes, what is its particular nature? What is the role of these structures in the general magnetospheric dynamics?

To this end, it should be noted that the complicated pattern of convection motions in the plasma sheet associated with magnetic field variations, occur on medium scales, large as compared to the plasma sheet thickness and small as compared to global magnetospheric dimensions. These motions are identified as a multitude of vortices, so together with corresponding magnetic variations, they may really be interpreted as manifestations of specific, basically twodimensional, MHD turbulence.

However, a number of unresolved questions exists, in relation to that turbulence. What does drive it? What is the energy source? In observations, bursty bulk flows (BBF) (e.g. Sergeev, 2004) may be the proper candidate. But what is their origin?

Other questions concern the mechanism of dissipation at the smallest scales if the energy presumably cascades from larger scales to smaller ones.

On the other hand, simultaneous appearance of a fast plasma flow and thin current sheets was described by the theory of the so called "reconnection layer" (Lin and Lee, 1994). The solution of the Riemann problem on decay of an arbitrary MHD discontinuity was extended to the case of collisionless plasma by means of numerical particle simulations using a hybrid code. For an initial configuration with a symmetric current layer and a zero guiding field, it appears that magnetic merging evolves, with formation of a fast,

Published by Copernicus Publications on behalf of the European Geosciences Union. 
Alfvénic flow between a pair of collisionless slow shocks. The shock fronts are kinetic, ion Larmor radius scale structures; on those structures, the magnetic energy brought from both sides to the central plane, is transformed into energy of the fast flow.

Note that another regime of magnetic field merging might involve the "forced current sheet (FCS)", a specific nonlinear embedded thin current structure with anisotropic ion flows. Inside the CS ions carry the current as they move along their Speiser orbits oscillating about the central plane, and forming a kinetic structure with ion Larmor radius transverse scale. Outside the CS they form pairs of counter-streaming flows along the field lines, with double-Alfvén speed shift between the flows. This stationary nonlinear plasma structure was recently studied in detail (Kropotkin and Domrin, 1995, 1996; Kropotkin et al., 1997; Sitnov et al., 2000).

In the most recent particle simulation carried out in our papers (Domrin and Kropotkin, 2003, 2004, 2007a,b,c), it was shown that another, quite different evolution path in dynamics of magnetic merging, that involves formation of FCS, is really possible. Which particular path is taken by the system, depends on the initial parameters.

Note however that the simulation results obtained in a model which is one-dimensional over spatial coordinates, have a limited meaning only, if taken as themselves. In these models, there is a weak departure from equilibrium. It is associated with a small nonzero magnetic field component perpendicular to the current plane which produces unbalanced magnetic tension along the CS. Just this departure initiates the process of magnetic field merging. We need to understand how can such quasi-one-dimensional, slightly out-ofequilibrium configurations appear during the overall evolution of the geomagnetic tail.

The task of this study is to analyse various phases of such an evolution in order to find out when and how do conditions arise for appearance of localized, medium-scale configurations with the above properties. We argue that this involves the global plasma sheet thinning associated in general with the magnetic flux "loading" process in the geomagnetic tail (Schindler, 1972; Birn and Schindler, 2002; Birn et al., 2006). Another important point is the two different time scales for MHD-type disturbances which occur in the tail lobes and in the plasma sheet. We point out the association of the former with very fast, explosion-type bursts of nonlinear tearing mode instability (Kropotkin et al., 2002a,b) which sporadically appear in the thinned plasma sheet. The latter time scale corresponds to the above magnetic field merging processes. On the basis of our earlier studies mentioned above, on structure and dynamics of extremely thin CS, we propose a scenario of inter-relation between medium-scale and kinetic-scale elements of the plasma sheet. Dynamical evolution of one-dimensional CS, with nonzero normal component of the magnetic field, produces not only extremely thin embedded current structures, but also fast plasma flows with velocity of the order of Alfvén speed. We argue that those flows may drive medium scale MHD turbulent motions, and discuss the possible feedback mechanism for generation of new bursts of magnetic field merging.

\section{Quasi-static adiabatic reconfigurations}

Studies by Birn and Schindler (see Birn et al., 2006, and references therein) have shown that quasi-static evolution of the geomagnetic tail obeying the adiabaticity condition, i.e. enthropy conservation in a flux tube, may lead to formation of extremely thin (in MHD - even singular) current sheets (CS). The analysis was based on asymptotic solution of the GradShafranov equation. It was proposed by Schindler for the situation where, in the 2-D plane geometry, with $\partial / \partial y=0$, the configuration scale over the x-direction, $L_{x}$, is much larger than that over the z-direction, $L_{z}$. The above quasi-static evolution results in appearance of CS fractions which are much thinner than $L_{z}$, with limiting thickness going to zero.

In the 2-D plane geometry, the isotropic plasma pressure is a function of a single nonzero component of the vector potential, $A_{y}=A: p=p(A)$. In the asymptotic solution, the pressure balance takes place:

$p(x, z)+\frac{B^{2}(x, z)}{8 \pi}=\hat{p}(x)$

where $\hat{p}(x)$ is the pressure on the central plane $z=0$. For the "closed" model, with $B_{n}=0$ at the magnetopause, the magnetopause position is presented with the following relation for its z-coordinate $a(x)$ :

$a(x)=\int_{A_{0}(x)}^{A_{b}} \frac{d A}{\sqrt{8 \pi[\hat{p}(x)-p(A)]}}$

where $A_{0}(x)$ is the vector potential at $z=0$, and $A_{b}$ is its value at the magnetopause. It is shown in Birn and Schindler (2002); Birn et al. (2006) that a particular sequence of equilibrium configurations may be identified if we fix some model relation between $a$ and $\hat{p}$, for example,

$a(\hat{p})=a_{m}+\left(a_{\infty}-a_{m}\right)\left(\left|\hat{p}-p_{m}\right| / p_{m}\right)^{k}$

Quasi-static transitions between equilibrium states belonging to the sequence, may be modeled if we further set the time dependence of the parameters, e.g. of the parameter $a_{m}$. Such a dependence may be uniquely defined if we assume that the transitions occur adiabatically, i.e. the pressure $p$ in the flux tube $A=$ const and its specific volume

$V(A)=\int_{0}^{x_{0}} \frac{d x}{B_{x}(x, A)}$

are related with the adiabatic equation, $p V^{\gamma}=$ const. And in this case it is possible to obtain such a sequence of locally thinning CS that the thickness goes to zero, and the current density goes to infinity. 
Let us point out that the same slow adiabatic evolution, but now considered on the global scale of the geomagnetic tail, should also maintain its basic feature: at every crosssection $x$, a dense and hot current-carrying plasma sheet exists in its central part, while a cold rarified plasma background exists outside it, in the tail lobes. Consider what is the density behavior in the tail configuration. The strong elongation of the tail means that over its particular crosssection $x$, those flux tubes $A=$ const which are outside CS, have much larger length, and correspondingly larger specific volume than those flux tubes which belong to the CS. On the other hand, the latter ones, at earlier time moments, had just such large length. This is because of the magnetospheric convection directed inwards, in the near-Earth part of the geomagnetic tail. The convection determines the flux tube evolution on average and on large time scales: they are shrinking, and the density is increasing. Thus the existence of a large pressure maximum near the central plane is evolutionally determined itself. It is a result of adiabatic compression accompanied by heating, which occurs in flux tubes convecting towards the Earth, and shrinking. This well known effect caused in the past a discussion on the so called "convection crisis". At a given cross-section $x$, those field lines which belonged initially to the tail lobes where $p \ll B_{0}^{2} / 8 \pi$ ( $B_{0}$ is the field intensity outside CS), are later transported inside CS. The pressure $p=p(A)$ of the plasma appearing inside CS, should be there equal to $B_{0}^{2} / 8 \pi$, i.e. to the magnetic pressure outside CS, as it follows from the balance of transverse momentum (Eq. 1). Therefore, during the convective transport, the pressure inside a particular flux tube strongly increases, as compared with its value at the time when the tube was still outside CS where $\beta=8 \pi p / B_{0}^{2} \ll 1$.

Thus the dominating on average, relatively slow and largescale evolution of the geomagnetic tail maintains the existence of a dense and hot plasma layer in its central part, in the plasma sheet, and of a cold rarified plasma background in the tail lobes.

Over this effect appear to be superimposed sporadic localized effects acting on medium scales, small as compared to the global magnetospheric dimensions, and large in comparison with the CS thickness. They involve CS thinning as it is discussed above.

\section{Fast reconfiguration with loss of equilibrium in the current sheet}

While considering large-scale processes of the magnetospheric-ionospheric dynamics, it is useful to separately identify processes of quasi-stationary and of transient nature. In Sect. 2 we treat the former ones. As to the latter ones, it is believed that during transition of the magnetospheric-ionospheric system from one stationary state into another, the latter establishes on the Alfvén time scale $\tau_{A}$. It is estimated as the transit time for the
Alfvén wave; with the magnetospheric Alfvén velocity $V_{A} \sim 1000 \mathrm{~km} / \mathrm{s}$, over a typical magnetospheric scale $\sim 10^{5} \mathrm{~km}$, this time is $\tau_{A} \sim 100 \mathrm{~s}$. Correspondingly, over an intermediate scale, of the order of a few $R_{E}$, the process duration is even much shorter, $\tau_{A} \sim 10 \mathrm{~s}$.

However, because of a specific feature of the magnetospheric configuration, namely the elongated geomagnetic tail with the plasma sheet separating its lobes, it would be inadequate to associate all the transient processes with a single time scale. Indeed, in the plasma sheet the magnetic field normal component $B_{n}$ is very small, and the Ampére force $\left[\mathbf{j B}_{\mathbf{n}}\right] / c$ inducing the plasma motion towards the Earth (or the outward one) is correspondingly small. Therefore such motions take relatively long time to appear, and this has a decisive influence upon those processes which involve such a motion. It follows that those processes may be considered separately, based on assumption that the previous evolution "abruptly" produces an out-of-equilibrium state of the magnetoplasma system, and that is followed by relatively slow relaxation. Note that this relates to such relaxation only, which involves the inward (outward) plasma motions in the plasma sheet. And such motions are a necessary constituent of the magnetic reconnection phenomenon in the geomagnetic tail.

The subsequent evolution of a configuration in which a thin CS has been formed on the preceding quasi-stationary stage, may be considered in a relatively simple manner if we take into account that outside the CS, "strong field" approximation (Syrovatsky, 1971) is valid:

$\beta=8 \pi p / B^{2} \ll 1 ; M_{A}=V / V_{A} \ll 1$

Then outside the CS the magnetic field is everywhere curlfree, and the Laplace equation

$\Delta_{2} A=0$

governs its potential $A$. The boundary condition may be taken in the form $\left.A\right|_{\Sigma}=f_{1}(s)$ where $s$ is a coordinate counted along the boundary $\Sigma$. Since the CS is thin, it is possible to consider a portion of the boundary of the strong field domain which is formed with its outer edge, to coincide with the $x$ axis.

Note that within the approximation (2) we may deal not only with a slow quasi-static evolution of the whole system. We may also consider such relatively fast variations which involve only that its part which is outside CS, so that this part has enough time for quasi-static restructuring. Indeed, formally such reconfigurations occur instantly, following the variation of the boundary conditions. In the Syrovatsky model, the speed of disturbance propagation tends to infinity, $V_{A} \rightarrow \infty$, and the potential $A$ at any moment, at any point is determined simply by solution of the corresponding boundary-value problem for the Laplace equation.

However from the physical point of view, the variation itself of the boundary conditions cannot be fully arbitrary. The boundary condition at $z=0$, i.e. the function $f_{1}(x, z=0)$ can 
change only slowly. Really, some fast variation in time of the potential $A=f_{1}(x, z=0)$ at a given point on the $z=0$ surface would mean an abrupt appearance of a fast MHD flow inside the CS itself, and that is impossible. We have shown above that as a result of preceding evolution, plasma inside the CS becomes relatively hot and dense: if the density outside CS is equal to $\rho_{1}$ and the mean density inside CS is $\rho_{0}$ then

\section{$\rho_{1} / \rho_{0} \ll 1$.}

It will now be shown that if there is a thin CS with all the characteristics varying along it on a large spatial scale, and it separates the two regions occupied with rarified cold plasma, then a flow has really no time to arise on the scale of fast variations in the system. So if the other boundary conditions really vary quickly, then everywhere outside CS the configuration varies but continues to be described by the Syrovatsky model, however with $f_{1}(x, z=0)$ remaining unchanged (on the time scale of those fast variations). And in the thin CS, the condition of force balance over the $\mathrm{x}$-direction is now violated. In that condition

$$
-\frac{\partial}{\partial x} \int_{-\infty}^{\infty} p d z+\frac{1}{4 \pi} B_{n} B_{0}=0
$$

the second term remains almost unchanged while the first one varies. Thus a loss of equilibrium takes place.

Let a region in the plasma sheet which is the location of an extreme thinning considered in Sect. 2, to have length $L$ over the x-direction. The scale $L$ is small in comparison to the global magnetospheric scale $L_{x}, L \ll L_{x}$. Outside CS the magnetic field reconfigures by means of propagating fast disturbances, with characteristic velocity equal to the Alfvén speed, $V_{A 1}=B_{0} / \sqrt{4 \pi \rho_{1}}$ (being though high, but finite velocity, see e.g. Domrin and Kropotkin, 2007a). Therefore the characteristic time $T_{1}$ which the reconfiguration takes to occur in the lobe region over the considered region, obeys the relation $L_{x} / V_{A 1} \gg T_{1} \gg L / V_{A 1}$. Since the corresponding wavenumber $k=\omega / V_{A 1}=2 \pi / V_{A 1} T_{1}$ is thus large enough so that $k L_{x} \gg 1$, such a disturbance may be described as a MHD wave in the WKB approximation, i.e. locally it is similar to the MHD wave disturbances on a homogeneous background. On the other hand, since

$k L=\left(\omega / V_{A 1}\right) L \ll 1$,

the magnetic field in the considered region, at every moment should obey equations of static field, i.e. the field is quasistationary. The field is so polarized that over the CS surface, (small) increases and decreases of the tangential component $\delta B_{t}$ appear. They produce small compressions and rarifactions in the CS, i.e. the similar fast changes in the parallel (in the x-direction) pressure distribution inside CS. Indeed, over the CS thickness $d \ll L$, that is very small as compared to the wavelength $2 \pi / k \gg L$, at every moment the transverse momentum component is balanced. Therefore the plasma pressure $p=p_{0}+\delta p$ at the central plane varies on the scale

$\delta p \sim\left[\left(B_{0}+\delta B_{t}\right)^{2}-B_{0}^{2}\right] / 8 \pi \simeq B_{0} \delta B_{t} / 4 \pi$.

Inside CS those variations produce parallel plasma motions. Let us estimate those variations. For a component with wavenumber $k$, the increment of the first term in Eq. (4) is $\sim(k d)(\delta p)_{k}$, and that of the second term is

$\sim \frac{1}{4 \pi}\left[B_{n}\left(\delta B_{t}\right)_{k}+B_{0}\left(\delta B_{n}\right)_{k}\right]$.

In a frame moving with the wave at the speed $V_{A 1}$, the CS is a stationary 1-D flow in a thin plane channel, with all the variables depending on a single parameter $x$. According to the Euler equation,

$\rho_{0} V_{A 1} \cdot \delta v(x)=-\delta p(x)$.

Since $V_{A 1}=/ \sqrt{4 \pi \rho_{1}}$ with the use of Eq. (6) we obtain

$\frac{(\delta v)_{k}}{V_{A 1}}=-\frac{B_{0}}{4 \pi \rho_{0} V_{A 1}^{2}}\left(\delta B_{t}\right)_{k}=-\frac{\rho_{1}}{\rho_{0}} \frac{\left(\delta B_{t}\right)_{k}}{B_{0}}$.

In a harmonic wave, in a half-period time a plasma element with length $\lambda / 2=\pi V_{A 1} / \omega$ stretches (or shrinks) by an increment $(\delta x)_{k} \sim \pi \cdot(\delta v)_{k} / \omega$. Under the disturbance action, because of the field frozen-in property, the field normal component $B_{n}$ will also vary, conserving the magnetic flux through the given plasma element. So the resulting relative decrease (increase) of the field is

$\frac{\left(\delta B_{n}\right)_{k}}{B_{n}}=\frac{2(\delta x)_{k}}{\lambda}=\frac{(\delta v)_{k}}{V_{A 1}}$.

A simple relation between relative variations of the normal and tangential field components and the relative pressure variation is thus obtained:

$\frac{\left(\delta B_{n}\right)_{k}}{B_{n}}=\frac{\rho_{1}}{\rho_{0}} \frac{\left(\delta B_{t}\right)_{k}}{B_{0}}=\frac{\rho_{1}}{\rho_{0}} \frac{(\delta p)_{k}}{2 p_{0}}$.

Estimate now the terms of Eq. (7). According to the balance condition (4) we have

$\frac{B_{n} B_{0}}{4 \pi} \sim p_{0} \frac{d}{L_{x}}$,

where $L_{x}$ is the parallel scale of the geomagnetic tail as a whole, i.e. the global magnetospheric scale. We point out that for our consideration, the natural hierarchy of scales is given by a double inequality

$L_{x} \gg 2 \pi / k \gg L$.

Based on Eq. (8), we obtain

$$
\begin{aligned}
\frac{1}{4 \pi} B_{n}\left(\delta B_{t}\right)_{k} & \sim p_{0} \frac{d}{L_{x}} \frac{\left(\delta B_{t}\right)_{k}}{B_{0}} \\
& \sim p_{0} \frac{d}{L_{x}} \frac{(\delta p)_{k}}{p_{0}} \sim \frac{d}{L_{x}}(\delta p)_{k} .
\end{aligned}
$$


For the second term we have

$$
\begin{aligned}
\frac{1}{4 \pi} B_{0}\left(\delta B_{n}\right)_{k} & \sim p_{0} \frac{d}{L_{x}} \frac{\left(\delta B_{n}\right)_{k}}{B_{0}} \\
& \sim p_{0} \frac{d}{L_{x}} \frac{\rho_{1}}{\rho_{0}} \frac{(\delta p)_{k}}{p_{0}} \sim \frac{d}{L_{x}} \frac{\rho_{1}}{\rho_{0}}(\delta p)_{k} .
\end{aligned}
$$

With $k L_{x} \gg 1$, the increment of the first term in the parallel momentum balance equation, $\sim(k d)(\delta p)_{k}$, much exceeds the increment (10). The increment (11) is in any case small, because the condition $\rho_{1} / \rho_{0} \lesssim 1$ is satisfied due to convective accumulation of plasma in the CS. So at $k L_{x} \gg 1$ the constituents (10) and (11) produce negligibly small corrections.

Now we propose that the disturbance magnitude, small as it is, is however large enough for the condition

$$
\left(k L_{x}\right) \frac{(\delta p)_{k}}{p_{0}} \gtrsim 1
$$

to be fulfilled. Such a disturbance violates the balance condition (4) considerably: locally, on a scale of the order of the wavelength $2 \pi / k$, and therefore on a smaller scale $L \ll 2 \pi / k$ as well, the initial parallel gradient may be considerably suppressed by that disturbance.

So on a short time interval $T_{1}$ the parallel pressure gradient in a localized region with size $L$ can be disturbed; then it is no more compensated by the magnetic tension (which varies only slightly), and the parallel equilibrium is violated. The time scale $T_{1} \sim 2 \pi / \omega=2 \pi / k V_{A 1}$ relates to wavelength $2 \pi / k$ intermediate between the global magnetospheric scale $L_{x}$ and the scale $L$, see Eq. (9).

The relaxation which follows, locally on the scale $L$ is determined by the small but nonzero value of the resulting parallel force. Because of its smallness, the relaxation occurs on a much longer time scale, $T_{2} \gg T_{1}$. The characteristic time $T_{2}$ of the nonlinear relaxation process may be approximately estimated based on the following argument. The new final balance of the parallel momentum is being reached by means of acceleration of ions in the plasma sheet by the electric field $E_{y}=E$ which has appeared,

$$
E=\frac{B_{n}}{c} V_{A 1}=\frac{B_{0} B_{n}}{c \sqrt{4 \pi \rho_{1}}}
$$

and of their rotation in the magnetic field $B_{z}=B_{n}$; after that the ion mean momentum becomes equal to $m_{i} V_{A 1}$ (see e.g. Domrin and Kropotkin, 2007a,b). So the momentum balance is given by condition

$\rho_{0} V_{A 1} d \sim \frac{B_{n} B_{0}}{4 \pi} T_{2}$,

It follows that

$T_{2} \sim \frac{\rho_{0}}{\rho_{1}} \frac{B_{0}}{B_{n}} \frac{d}{V_{A 1}} \sim \frac{\rho_{0}}{\rho_{1}} \frac{L_{x}}{V_{A 1}}$

since in the initial equilibrium we have

$$
\frac{d}{L_{x}} \sim \frac{B_{n}}{B_{0}}
$$

On the other hand, $T_{1} \sim 2 \pi / k V_{A 1}$ so that because of smallness of the parameters $2 \pi / k L_{x} \ll 1$ (see Eq. 9) and $\rho_{1} / \rho_{0} \ll 1$ (Eq. 3), the inequality $T_{2} \sim\left(\rho_{0} / \rho_{1}\right)\left(k L_{x} / 2 \pi\right) T_{1} \gg T_{1}$ is really valid. And with such large difference of the time scales $T_{1}$ and $T_{2}$, the simplest model of the process will be relaxation of a 1-D layer with uniform pressure over the xdirection but with an unbalanced magnetic tension abruptly appearing at the initial moment $t=0$. In other words, the model system is "prepared" at the moment as a 1-D system, being out of equilibrium because of the magnetic field nonzero component $B_{n}$.

To resume, a strong difference between the parallel and transverse spatial scales, together with the relatively high plasma density inside the CS, which develop at a slow adiabatic reconfiguration involving strong thinning of the CS that is described in the Schindler model, allows to introduce an important simplification into the analysis. Fast reconfigurations of the outer region (the tail lobes) may be described by the Syrovatsky model. In the plasma sheet they "instantly" generate localized pressure nonuniformities, and corresponding violations of the parallel force balance.

\section{Relaxation of the quasi-one-dimensional thin CS and magnetic field merging}

Study of the relatively slow relaxation process on the time scale $T_{2}$ was carried out in Domrin and Kropotkin (2003, $2004,2007 \mathrm{a}, \mathrm{b}, \mathrm{c})$, and it will be briefly outlined here. Underlying assumption is that at some locations on the thin CS, abrupt losses of equilibrium occur, and they are later followed with much slower relaxation of the out-of-equilibrium system thus arising. In earlier simulation (Lin and Lee, 1994), such an evolution could involve the Hall effect and generation of magnetic field $\mathrm{y}$-component, which produces formation, among others, of rotational discontinuity-like structures. However, at $\rho_{1} / \rho_{0} \ll 1$, the CS evolution is slow enough that the Hall effects may be neglected (see Domrin and Kropotkin, 2007c). Since the appearing thin CS's have thickness on the order of the ion gyroradius, significant new features appear in the process due to kinetic effects. Their role in this nonlinear time-dependent problem has been identified by means of simulation with the use of a particle (hybrid 1-D) code. It starts with a slightly outof-equilibrium state with a small $B_{n}$ and correspondingly, a small unbalanced magnetic tension. It turns out that the process may follow one of the two quite different evolution paths. Both relate to the nonlinear dynamics of thin CS. It is found out that the dynamics always involves features of SELF-ORGANIZATION: thin EMBEDDED current structures spontaneously appear and then exist for a long time, with very high current density inside. One of these two paths is a kinetic version of the well known model of arbitrary discontinuity decay (the Riemann problem in MHD). From the initial CS, a pair of fast magnetosonic rarefaction waves and 


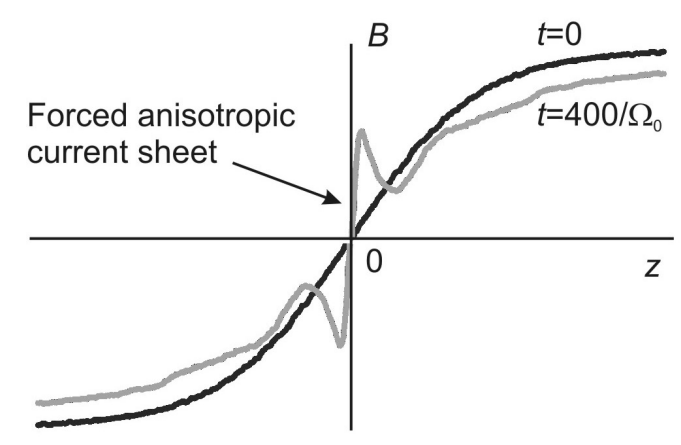

Fig. 1. Examples of the simulation results: calculated field profiles.

a pair of slow switch-off shocks are born, propagating to both sides from the location of their origin. At the shock fronts energy transformation takes place, and a fast plasma flow appears between them. During the process which develops spontaneously, a finite relaxation rate maintains, nearly independent of time. The intensity of the electric field (Eq. 12) which establishes, has been calculated, as well as the electromagnetic energy flux

$P=c \frac{E B_{0}}{4 \pi}=\frac{B_{0}^{2} B_{n}}{(4 \pi)^{3 / 2} \sqrt{\rho_{1}}}$

into the central region where the energy is transformed into kinetic energy of a (fast) plasma flow.

Another evolution path leads to formation of a specific kinetic one-dimensional structure located in the vicinity of the central plane. The structure also appears to be quasistationary. It possesses the properties of the kinetic forced current sheet (FCS), with strongly anisotropic ion distributions. This is a special kinetic solution which was found out and studied in detail in the papers (Kropotkin and Domrin, 1995, 1996; Kropotkin et al., 1997; Sitnov et al., 2000). In this case also, fast ion flows are formed. The counterstreaming ion distributions appear to be unstable; they should generate plasma wave turbulence which eventually produces ion scattering, and energy dissipation.

The clue parameter is the value of magnetic field normal component $B_{n}$. There is a critical value $B_{n}^{(c r)}$ that separates the regions where the above two regimes take place. At relatively high $B_{n}>B_{n}^{(c r)}$, the embedded thin current structures have the characteristics of switch-off shocks. At $B_{n}<B_{n}^{(c r)}$ a thin structure of the anisotropic FCS is formed in the central region. In both cases, at the late stages the process appears to be self-sustained, independent of the initial disturbance type; the disturbance intensity SPONTANEOUSLY establishes at a finite level determined by relations (12-13). Two typical examples of the magnetic field profile involving kinetic thin CS are shown in Fig. 1.

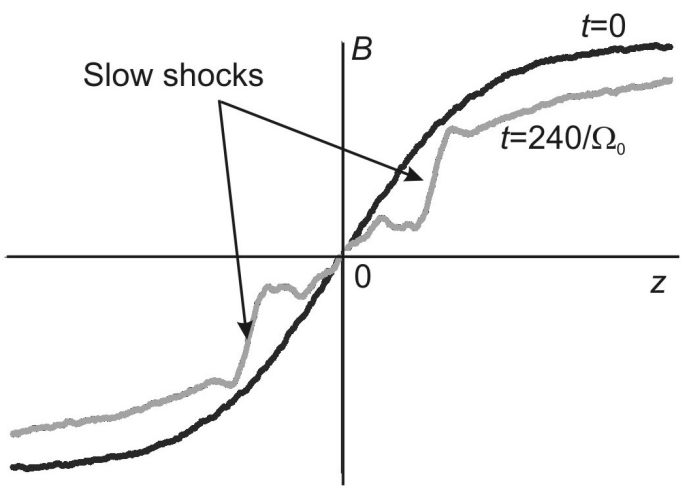

We thus obtained two versions of "reconnection layer" (Lin and Lee, 1994) formation. At the medium scale level in the geomagnetic tail, this dynamics presents spontaneous process of magnetic field merging; the magnetic field flux and energy are brought inside by a pair of MHD self-similar rarefaction waves propagating over the background plasma from the nonlinear structure which has formed.

Ion distribution functions have been obtained. They have generally complicated anisotropy. Examples of distribution functions are demonstrated in Fig. 2. The red bar denotes the value of the hot ions thermal velocity (it is equal to the Alfvén velocity outside the plasma sheet, which follows from the transverse momentum balance), the green one - that of the cold ions. The distributions show different anisotropy features: fast bulk flows (plot d), double-humped distributions of counter-streaming flows (plot c), patchy distribution formed by ions on their Speiser orbits near the central plane (plot b).

\section{Initiation of fast reconfiguration - inter-relation of various type disturbances in the plasma sheet}

At present there is no comprehensive understanding of the cause of abrupt reconfigurations. The simplest possibility is associated with fast variations of the magnetopause under the action of a disturbance in the solar wind flow past the magnetosphere. Another possibility is associated with an internal cause. Based on the available theoretical results, a scenario may look as follows. Locally, in some near-Earth portion of the plasma sheet, during the quasi-static evolution of the system, accompanied by CS thinning (Sect. 2; see also Birn et al., 2006, and references therein), the state of marginal stability is reached, relative to tearing-mode disturbance (or to the ballooning mode disturbance, see e.g. Hurricane et al., 1999). This is followed by a nonlinear effect of "pulling" of the system into the zone of (linearly) unstable equilibrium, and later, by a fast, abrupt loss of equilibrium, the "jump" of the system 

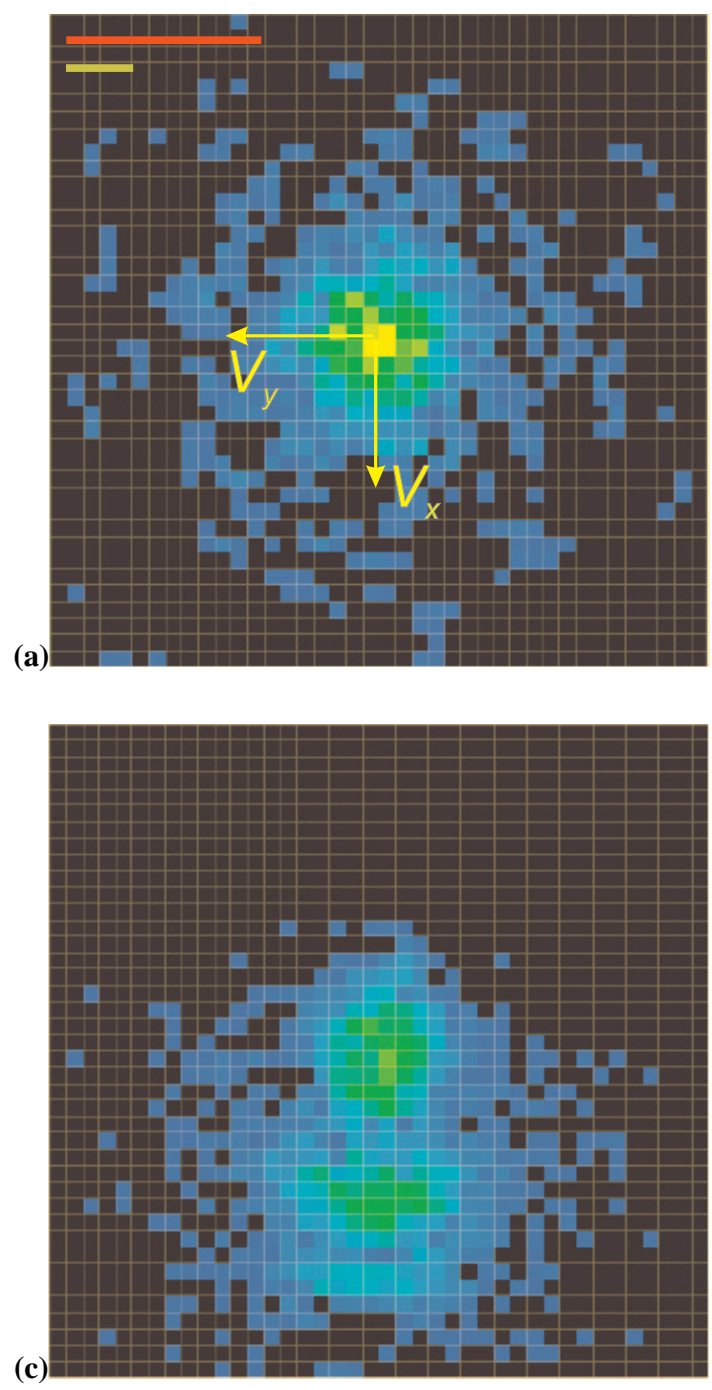

(b)
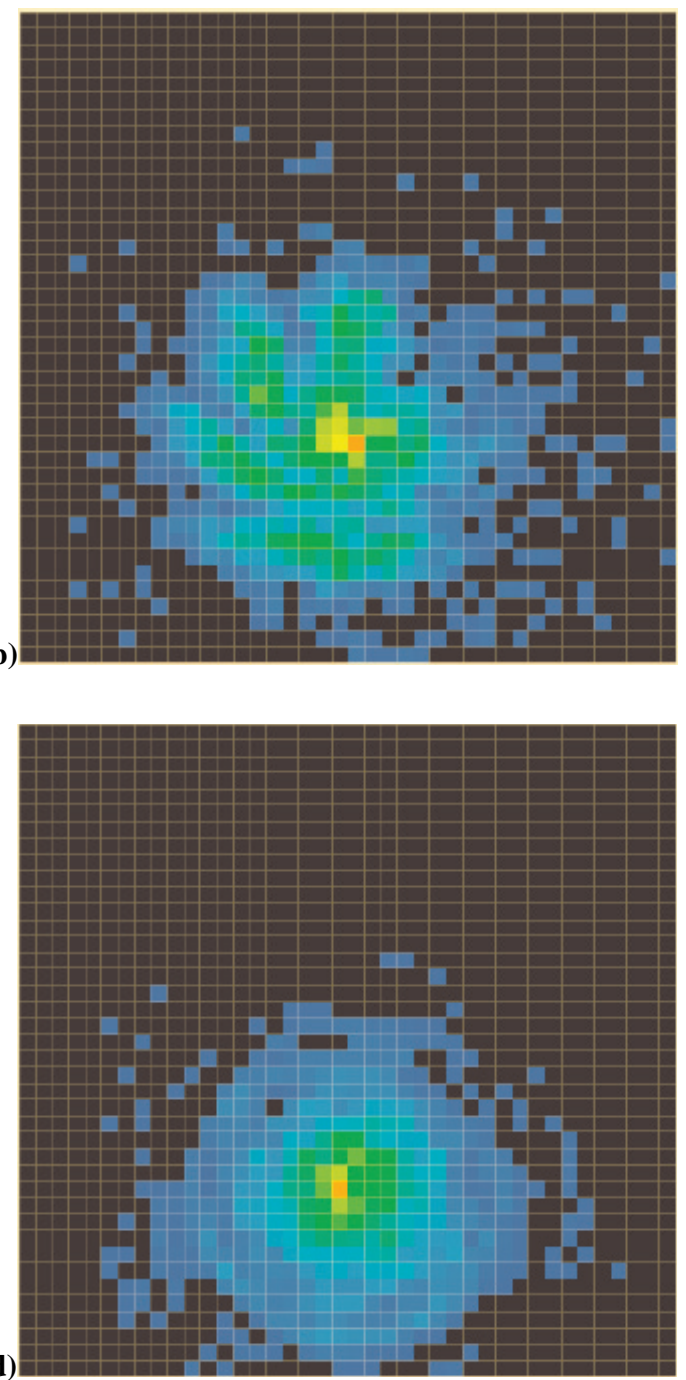

Fig. 2. Examples of distribution functions: phase space densities at $v_{z}=0 ; v_{x}=v_{y}=0$ at the center of each plot. (a) Initial state at $B_{n} / B_{0}=0.12, z=0$. (b) Later moment $t=4 / \Omega_{0} ; B_{n} / B_{0}=0.12, z=0$. (c) $t=4 / \Omega_{0} ; B_{n} / B_{0}=0.12, z=12.2 \rho_{0} . \quad\left(\right.$ d) $t=4 / \Omega_{0} ; B_{n} / B_{0}=0.2$, $z=0$. The red bar is the value of the hot ions thermal velocity, the green one - that of the cold ions. Density decrease is denoted by the color sequence, from red to blue; black denotes negligible density.

onto a new equilibrium state (topologically different because of the magnetic reconnection) (Kropotkin et al., 2002a,b).

While the tearing mode in 1-D geometry is evanescent, i.e. decays rapidly in the $\mathrm{z}$-direction, however in the geomagnetic tail, the tearing-like disturbances should be nonuniform, localized over $x$ and $y$. Because of this, the tearing mode appears to be linearly coupled to MHD waves. The MHD waves are propagating; they transport outside CS the jump of the magnetic flux and magnetic field values, which are caused by the magnetic reconnection burst occurring in the region of the nonlinear tearing instability action, inside CS and in its vicinity. The disturbance is transferred along the geomagnetic tail, over the cold rarified plasma of its lobes, with high velocity $V_{A 1}$.
A fast reconfiguration arises in the lobes, involving also regions distant from the zone of action of initiating tearing instability; so the out-of-equilibrium portions of CS are being "prepared" (Sect. 3). This sequence of events is sketched in Fig. 3.

The well known models of magnetic reconnection based on kinetic simulations, e.g. those in the series of papers opening with the paper (Birn et al., 2001), present the system dynamics in the vicinity of an already existing magnetic field neutral line. On the other hand, as it was shown in a number of papers based on that same numerical simulation, e.g. in Kuznetsova et al. (2001, 2007), in that subsequent evolution of the configuration, the reconnection rate at the neutral line is itself adjusting to the large scale (medium scale - in our 


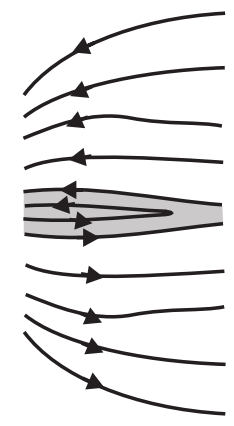

(a)

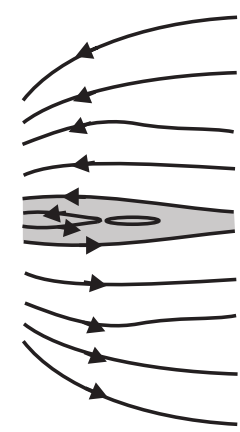

(b)

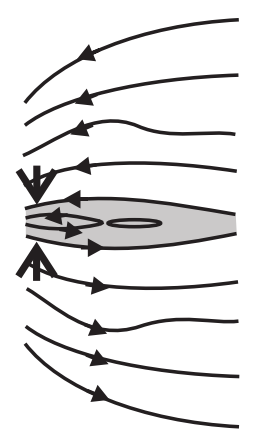

(c)
Fig. 3. (a) Equilibrium thin plasma sheet with current-carrying dense hot plasma, on a uniform cold low density background. (b) The sheet after a localized burst of nonlinear tearing instability - impulsive reconnection. The topology has changed: a magnetic island has appeared. (c) CS thinning by means of a MHD pulse escaping the reconnection zone over the plasma of tail lobes. The equilibrium in $\mathrm{x}$-direction in the thinning zone is violated.

case) dynamics. Namely the characteristics of that dynamics are the subject of our study. The above rate is locally determined by the rate of energy transformation, which in its turn is governed by the value of magnetic field energy flux towards the CS (Eq. 13).

As it was mentioned in the Introduction, in observational data obtained recently onboard spacecraft in the geomagnetic tail, nonlinear non-stationary structures of the two quite different types were identified. On the one hand, very thin current sheets sporadically appear in the plasma sheet, embedded in its thicker plasma structure. And on the other hand, at those same locations, at intermediate scales of the order of a few $R_{E}$, strong magnetic variations, along with accompanying plasma motions, were observed.

Based on the above argument, it may be proposed how can the two types of disturbance be inter-related, and can form a unified pattern of specific turbulence in the plasma sheet.

Simultaneously with embedded thin current structures, ion flows of two different types appear: first, a flow escaping the CS with the doubled Alfvén speed; second, a flow with mean velocity equal to the Alfvén speed, which remains inside the CS and in its nearest vicinity, and moves earthwards (or backwards). Since these fast flows arise on a large time scale $T_{2}$, themselves and their accompanying effects have predominantly quasi-stationary character. In observations, they may be visualized, first, as quasi-stationary parallel fast ion flows (beamlets) in the auroral magnetosphere, and second, as quasi-stationary convection jets, with corresponding electric and magnetic fields. Since the disturbed zone of the CS where the relaxation takes place, has a limited size over the direction $y$ as well as over $x$, just localized jets appear: quickly shrinking flux tubes penetrate the neighboring ones which are in nearly equilibrium, static condition.
However, in addition to the quasi-stationary features, there are also fast, wave constituents of the disturbance. The fast MHD rarefaction waves propagating outwards from the $\mathrm{CS}$, on the scales exceeding the disturbance wavelength, $\gtrsim V_{A 1} T_{1} \gg L$ (see Eq. 5), transfer the electric field of the disturbance into the neighboring zones. That produces there plasma sheet thinning which occurs in a quasi-stationary manner: its rate at a given point grows slowly, following the $E$ change occurring on the time scale $T_{2}$. So these reconfigurations occur simultaneously on a large scale $\sim V_{A 1} T_{2}$.

Such a thinning may locally produce a new nonlinear tearing instability and corresponding process of fast reconnection, in a new limited region. The subsequent events follow the above scenario. The pattern may be more complicated because of fast MHD disturbances appearing as waves reflected from the ionosphere.

Locally generated fast ion flows may support in the plasma sheet the turbulent cascade of diminishing in scale, nonstationary two-dimensional vortical motions, and the associated magnetic field variations. Those slow motions, with their corresponding electric fields, are not related to MHD wave disturbances, unlike the kinetic thin CS structures, see above, so the two might look unrelated. Note however that turbulent plasma motions driven by such a flow, may be an agent of internally driven localized (contrary to the wavedriven mechanism, see above) plasma sheet thinnings followed then by new bursts of the nonlinear tearing instability. New processes of dynamical evolution should result, leading to new extremely thin CS formation. These effects are proposed to be the basic ones for the overall magnetospheric dynamics.

\section{Conclusions}

Processes of complicated dynamics of the geomagnetic tail involve cycles of electromagnetic energy loading and unloading, fast losses of equilibrium, embedded thin CS, medium scale turbulent plasma motions and intense magnetic field variations. Conceivably, the processes are interrelated. A plausible scenario of such inter-relation is proposed in this paper. Basically, in the tail dynamics, a fast mechanism should operate which sporadically dissipates the magnetic field energy, transforming it into energy of plasma flows, and heat. That occurs in those regions where $(\mathbf{j E})>0$ the reconnection layers, i.e. in the regions which (a) are occupied with a current sheet (CS), and (b) have the electric field $\mathbf{E}$ penetrated into the CS. For this reason, the central role is attributed here to nonlinear dynamical processes of thin kinetic CS formation associated with induced electric field appearance. Embedded nonlinear current structures of a reconnection layer appear as a result. They turn out to be quasi-stationary; they involve the nonzero field $\mathbf{E}$, and so are responsible for fast transformation of magnetic field energy into energy of plasma flows. The latter, in their 
turn, can be the drivers of the two-dimensional MHD turbulence in the plasma sheet. While the spontaneously arising thin current structures are quasi-stationary, at large spatial scales the disturbance remains wave-like: it involves the fast magnetosonic constituent. The latter may serve as an agent inducing new plasma sheet thinnings; turbulent vortical motions may be an additional agent. These thinnings are again followed by losses of parallel equilibrium in new localized zones of the plasma sheet, and then - by relaxation processes, with formation of embedded thin CS and fast ion flows. The intermittency which thus arises, is presumably a characteristic feature of turbulent disturbances being observed in the plasma sheet.

Acknowledgements. This work was supported by the Russian Foundation for Basic Research (project no. 05-05-64993).

Topical Editor R. Nakamura thanks A. Klimas and another anonymous referee for their help in evaluating this paper.

\section{References}

Birn, J. and Schindler, K.: Thin current sheets in the magnetotail and the loss of equilibrium, J. Geophys. Res., 107A, 1117, doi:10.1029/2001JA000291, 2002.

Birn, J., Drake, J. F., Shay, M. A., Rogers, B. N., Denton, R. E., Hesse, M., Kuznetsova, M., Ma, Z. W., Bhattacharjee, A., Otto, A., and Pritchett, P. L.: Geospace Environmental Modeling (GEM) magnetic reconnection challenge, J. Geophys. Res., 106A3, 3715-3719, 2001.

Birn, J., Hesse, M., and Schindler, K.: Modeling of the magnetospheric response to the dynamic solar wind, Space Sci. Rev., 124, 103-116, 2006.

Borovsky, J. E. and Funsten, H. O.: MHD turbulence in the Earth's plasma sheet: Dynamics, dissipation, and driving, J. Geophys. Res., 108A, 1284, doi:10.1029/2002JA009625, 2003.

Domrin, V. I. and Kropotkin, A. P.: Kinetic current sheet origination in the geomagnetic tail: simulation using particles, Geomagn. and Aeron, (English translation), 43, 276-283, 2003.

Domrin, V. I. and Kropotkin, A. P.: Forced current sheet structure, formation and evolution: application to magnetic reconnection in the magnetosphere, Ann. Geophys., 22, 2547-2553, 2004, http://www.ann-geophys.net/22/2547/2004/.

Domrin, V. I. and Kropotkin, A. P.: Dynamics of equilibrium upset and electromagnetic energy transformation in the geomagnetotail: a theory and simulation using particles. 1. Evolution of configurations in an MHD approximation, Geomagn. Aeron. (English translation), 47, 299-306, 2007a.

Domrin, V. I. and Kropotkin, A. P.: Dynamics of equilibrium upset and electromagnetic energy transformation in the geomagnetotail: a theory and simulation using particles. 2. Numerical simulation using particles, Geomagn. Aeron. (English translation), 47, 307-315, 2007b.
Domrin, V. I. and Kropotkin, A. P.: Dynamics of equilibrium upset and electromagnetic energy transformation in the geomagnetotail: a theory and simulation using particles. 3. Versions of formation of thin current sheets, Geomagn. Aeron. (English translation), 47, 555-565, 2007c.

Hurricane, O. A., Fong, B. H., Cowley, S. C., et al.: Substorm detonation, J. Geophys. Res., 104A, 10 221-10 231, 1999.

Kropotkin, A. P. and Domrin, V. I.: Ion dynamics and induced reconnection in a thin current sheet, Geomagn. Aeron., 35, 1-7, 1995 (in Russian).

Kropotkin, A. P. and Domrin, V. I.: Theory of a thin onedimensional current sheet in collisionless space plasma, J. Geophys. Res., 101A, 19893-19 902, 1996.

Kropotkin, A. P., Malova, H. V., and Sitnov, M. I.: The selfconsistent structure of a thin anisotropic current sheet, J. Geophys. Res., 102A, 22 099-22 106, 1997.

Kropotkin, A. P., Trubachev, O. O., and Schindler, K.: Nonlinear mechanisms for the substorm explosion in the geomagnetic tail, Geomagn. Aeron. (English translation), 42, 277-285, 2002a.

Kropotkin, A. P., Trubachev, O. O., and Schindler, K.: Substorm onset: fast reconfiguration of the magnetotail caused by explosive growth of the turbulence level, Geomagn. Aeron. (English translation), 42, 286-294, 2002b.

Kuznetsova, M. M., Hesse, M., and Winske, D.: Collisionless reconnection supported by nongyrotropic pressure effects in hybrid and particle simulations, J. Geophys. Res., 106A, 3799-3810, 2001.

Kuznetsova, M. M., Hesse, M., Rastatter, L., et al.: Multiscale modeling of magnetospheric reconnection, J. Geophys. Res., 112, A10210, doi:10.1029/2007JA012316, 2007.

Lin, Y. and Lee, L. C.: Structure of reconnection layer in the magnetosphere, Space Sci. Rev., 65, 59-179, 1994.

Runov, A., Sergeev, V. A., Nakamura, R., et al.: Local structure of the magnetotail current sheet: 2001 Cluster observations, Ann. Geophys., 24, 247-262, 2006, http://www.ann-geophys.net/24/247/2006/.

Schindler, K.: A self-consistent theory of the tail of the magnetosphere, Earth's Magnetospheric Processes, edited by: McCormac, B. M., 200-210, D. Reidel, Norwell, Mass., 1972.

Sergeev, V. A.: Bursty bulk flows and their ionospheric footprints, Multiscale processes in the Earth's magnetosphere: from Interball to Cluster, edited by: Sauvaud, J. A. and Nemecek, Z., Kluwer, Netherlands, 289-306, 2004.

Sitnov, M. I, Zelenyi, L. M., Malova, H. V., and Sharma, A. S.: Thin current sheet embedded within a thicker plasma sheet: Selfconsistent theory, J. Geophys. Res., 105A, 13 029-13 043, 2000.

Syrovatsky, S. I.: On generation of a current sheet in a plasma with frozen-in magnetic field, Soviet JETP, 60, 1727-1741, 1971 (in Russian).

Weygand, J. M., Kivelson, M. G., Khurana, K. K., et al.: Plasma sheet turbulence observed by Cluster II, J. Geophys. Res., 110, A01205, doi:10.1029/2004JA010581, 2005. 